Original Research Paper

\title{
Immunological Response of Albino Rats Immunized with UV- Killed Candida albicans
}

\author{
${ }^{1}$ Ahmad Ibrahim, ${ }^{1}$ Yahaya Abdullahi Umar and ${ }^{2}$ Mohammed Shehu Busu \\ ${ }^{I}$ Department of Biological Sciences, Nigerian Defence Academy, Nigeria \\ Department of Microbiology and Biotechnology, \\ ${ }^{2}$ National Institute of Pharmaceutical Research and Development, Abuja, Nigeria
}

Article history

Received: 20-04-2019

Revised: 26-05-2019

Accepted: 08-07-2019

Corresponding Author:

Ahmad Ibrahim

Department of Biological

Sciences, Nigerian Defence

Academy, Nigeria

Email: ahmadibrahim337@yahoo.com

\begin{abstract}
The treatments of systemic candidiasis in humans with the current antifungal drugs of choice such as azole derivatives and amphotericin B have raised issues of toxicity and resistance among others. The immunogenic property of killed C. albicans was explored to evoke immunological response in the possible prevention of candidemia. Rats were immunized after acclimatization on two occasions with $10^{6}$ cells $/ \mathrm{ml}$ of Ultraviolet radiation killed $C$. albicans and subsequently challenged with $10^{6}$ viable $C$. albicans. Two other groups; positive and negative controls received same dose of viable $C$. albicans and normal saline respectively. Five rats each from the groups mentioned were bled for the determination of antibody titers and white blood cell differentials. Also, other rats from the three groups were observed for 28 days for survival after challenge to determine the protective effect of the ultraviolet killed $C$. albicans. The results revealed that Ultraviolet killed $C$. albicans stimulated a significant amount of antibodies and white blood cell differentials that were immunoprotective. We also found that all immunized rats survived challenge with $10^{6}$ viable cells while the kidneys of the dead unimmunized rats showed a positive growth of $C$. albicans. Thus, Ultraviolet killed $C$. albicans could provide significant protection.
\end{abstract}

Keywords: Candida Albicans, Candidiasis, Immunoprotection, Radiation

\section{Introduction}

It is well known that vaccines are developed for the control or prevention of diseases. They are derived from cell surface molecules (proteins and carbohydrate moieties), engineered organisms that have lost their virulence and pathogenecity or even killed organism that are immunogenic or have the ability of stimulating immune responses against a specific disease causing microorganisms (Evron, 1980). The cell wall of microbes acts as protective, structural shield and also contributes to interactive contacts with the human host during the initiation of infection and development (Tada et al., 2013).

Candida albicans is the most common candida species that causes candidiasis or yeast infection (CDC, 2016). It is reported to be present in $80 \%$ of human population as commensal but also has the ability of transiting to pathogenic forms when there is an imbalance of the normal microbiological flora, breakage of epithelial barriers or dysfunction of the immune system (Staib and Morschhäuser, 2007; Gow and Hube, 2012). These conditions may result from a wide-broad spectrum use of antibacterial, surgery, mutation or heredity among other factors (Spellberg et al., 2012). Candida albicans have been reported to cause two major types of infection; viz: superficial and disseminated (systemic) infection (Ortega et al., 2010). Superficial infection in candidiasis is usually localized around the skin, mouth and genitals whereas in systemic infection C. albicans gained access to the blood stream and invade the organs (Ortega et al., 2010). Symptoms associated with superficial candidiasis include discharge, itching and inflammation of the skin while in systemic infection; kidney or brain damage may occur (Leleu et al., 2002). According CDC, $75 \%$ of women with vulvo-vaginal candidiasis have been reported to have at least one episode of candidiasis while $40-45 \%$ has recurrent episodes (CDC, 2015). In addition, candidemia which is a life threatening infection accounts for $50-70 \%$ of all cases of invasive mycoses (Lamet al., 2016). Despite the achievements recorded in the treatment of systemic infection, approaches still depend on the use of chemotherapy (antifungal agents). Therefore, search for novel prophylactic alternatives that is effective and safe 
with no side effects continues due to a number of limitations with existing antifungal drugs such as toxicity, high cost and most times not available (Wang et al., 2015). More disturbing is the emergence of resistance to most of the commonly used antifungal drugs (Wang et al., 2015). This has led to increasing interest in the search for alternative strategies with immunostimulatory properties from a killed organism, subunits vaccine and even attenuated organisms for the protection of the host. Researchers mostly from other countries have reported the immunostimulatory potentials of $C$. albican killed using heat method with a number of solvent or adjuvants in mice (Evron, 1980; Lopez-Ribota et al., 2004; Cárdenas-Freytaget al., 1999). However, past studies in Nigeria on development of prophylactic options for the prevention of systemic candidiasis is scanty. Owing to the prevalence of mortality and morbidity attributed to systemic infection, it has become necessary to initiate studies. Therefore, the objective of this study therefore is to explore the potential of Ultraviolet (UV) radiation killed C. albicans sensitized host immune cells for the prevention of $C$. albicans infection.

\section{Materials and Methods}

\section{Experimental Animals}

Male albino rats of 6-7 weeks old were used in this study. The rats were provided with water and food ad libitum and allowed to acclimatize for 7 days prior to the commencement of the experiment. The use of animal for this study was approved by the Department of Pharmacology and Toxicology, National Institute of Pharmaceutical Research and Development on the standard practices of handling experimental animals in line with international best practice.

\section{Vaccine Preparation}

Candida albicans isolate was obtained from a clinical sample of a patient suspected with candida vaginitis. An informed consent was sought from the patient before the collection of the sample. The sample was cultured on Sabouraud Dextrose Agar (SDA) at $37^{\circ} \mathrm{C}$ for $2 \mathrm{~h}$ (Pereira et al., 2011). The creamy, white and pasty appearance of $C$. albicans produced on SDA was subcultured on a chromogenic agar (colour based selective medium) at $37^{\circ} \mathrm{C}$ for $48 \mathrm{~h}$ (Cooke et al., 2002). This was followed by a germ- tube test to differentiate albicans from non- albicans. Germ tube test was achieved by inoculation of a portion of $C$. albicans in serum and incubated at $37^{\circ} \mathrm{C}$ for $3 \mathrm{~h}$. This preparation was viewed under the microscope for the presence of filament or outgrowth (Elmer et al., 1992). Candida albicans used for immunization was exposed directly to a source of UV (Vilber Lourmat, UV trans-illuminator) at a wavelength of $254 \mathrm{~nm}$. Non-viability was confirmed every 10 minutes using methylene blue stain and subculturing on SDA for 30 min (Evron, 1980).

\section{Immunization and Challenge}

Three types of experiments were carried out in this study. Hence, rats were divided into fifteen each of three groups namely; negative control, positive control and UV inactivated group. Baseline was taken for all the rats 7 days after acclimatization. The UV inactivated group was immunized with $10^{6}$ UV killed $C$. albicans subcutaneously on two occasions 7 days after acclimatization and 21 days after. Positive and negative controls received same concentration and route of viable cells and sterile normal saline respectively on the $21^{\text {st }}$ day. This is followed by an intravenous (caudal) challenge with viable $C$. albicans after 14 days of booster immunization.

The first experiment was performed to determine variation or abnormalities of antibody titers and white blood cells differentials. Five rats each from the three groups were finally sacrificed after 3 days of challenge and their blood collected by cardiac puncture. The following parameters were determined using relevant assays as detailed below to check abnormalities.

\section{Determination of WBC Differentials and Antibody Titers}

Blood samples were collected in EDTA bottles and analyzed with the aid of an automated hematology analyzer (Abacus 380) to determine the frequency of abnormalities of white blood cell differentials.

Antibody titers were determined as described by BinHafeez et al. (2001). Blood samples were collected in plane bottles and centrifuged at $2500 \mathrm{rpm}$ for 10 minutes and sera obtained using a micropipette. As much as 100 $\mu \mathrm{l}$ of serum was heat- inactivated at $56^{\circ} \mathrm{C}$ in water bath for 30 minutes. About $50 \mu \mathrm{l}$ of Phosphate Buffered Saline (PBS) was then added to all 12 tubes. The first tube was considered the control hence it only received 50 $\mu \mathrm{l}$ of PBS, while the second well received $50 \mu \mathrm{l}$ of heatinactivated serum to form a mixture of serum and PBS. From the second tube, $50 \mu \mathrm{l}$ of the mixture was used to serially dilute by 2 - fold in the subsequent tubes. Finally, $50 \mu \mathrm{l}$ of heat killed $C$. albicans with a cell density of $10^{6}$ cells $/ \mathrm{ml}$ was added to all the tubes and incubated at $37^{\circ} \mathrm{C}$ for $2 \mathrm{~h}$. The values of antibody titer were assigned to the highest serum dilution showing at least $50 \%$ of visible agglutination.

\section{Determination of Weekly Survival Analysis}

The second experiment was carried out to determine survival of the rats after challenge. This was achieved according to Thomas et al. (2006) method. Another five rats each from the three groups that were immunized and challenged as described above were not sacrificed but monitored for a period of 28 days after challenge. Kidneys of dead rats during this period were harvested. The kidneys were sectioned, stained with crystal violet stain (microscopic stain BDH chemicals Ltd) and viewed 
under $\times 500$ digital microscope (Coolinttech, UK). Also homogenized portion were cultured on agar plate containing chloramphenicol. This is to check for the growth of C. albicans.

\section{Compliance with Ethical Standards}

There is no conflict of interest. The patient was also consented before the sample was taken for identification. The use of rats in this study is in accordance with the international best practices approved by the department of Pharmacology and Toxicology, National Institute of Pharmaceutical Research and Development, Abuja.

\section{Statistical Analysis}

The data collected from this study were subjected to statistical analysis. Analysis of Variance (ANOVA), student t-test and simple percentage were used to analyze these parameters. Analyses were performed using VassarStat Software (USA). The level of significance was set at a $P$ value of $<0.05$.

\section{Results}

\section{Identification of Candida Albicans}

Candida albicans isolates from the clinical sample obtained produced green coloration on the selective medium of Brilliance Candida $\mathrm{Agar}^{\mathrm{TM}}$, germ tubes (small, sprouting tube-like outgrowths filaments projecting from the cell surface) were formed in serum on incubation at $37^{\circ}$ and also a white to cream in color with smooth border appearance was formed on SDA plate incubated at $37^{\circ} \mathrm{C}$ to confirmed C. albicans. Cells exposed to UV radiation became stained in methylene blue and showed no evidence of growth on SDA plates. This confirms non- viability of the cells.

\section{Antibody Titers of Immunized Rats and Controls}

Result of antibody titers of rats showed that vaccination with UV killed $C$. albicans stimulated significant $(\mathrm{P}<0.05)$ amount of antibodies compared to controls (Fig. 1). The mean baseline antibody titer of the rats taken after 7 days of acclimatization was $25.6 \pm 10.55$ for all the groups. After two occasions of vaccinations (first and booster vaccinations) and challenge, mean antibody titers recorded for the negative control group increases slightly to $32 \pm 8.76$ from the baseline titers of $25.6 \pm 10.55$.

The difference between antibody titers of rats in the negative control when compared to the baseline titers did not show any statistically significant difference $(\mathrm{P}>0.05)$. However, in the UV treated group, there was a statistically significant $(\mathrm{P}<0.05)$ increase in antibody titers $(972.8 \pm 307)$ when compared with both control groups after first and booster immunizations with UV killed $C$. albicans and subsequent challenge with viable cells. The positive control which was not immunized but infected during the same period of the study showed a statistically significant $(\mathrm{P}<0.05)$ lower mean antibody titers $(256 \pm 70.11)$ when compared to the immunized group.

\section{WBC of Immunized Rats and Controls}

The WBC count of albino rats vaccinated and challenged with $C$. albicans is presented in Fig. 2. The mean WBC count of the rats after acclimatization (baseline) was 7.6 \pm 0.3195 . At the end of the experiment, the mean WBC counts of rats in the negative control group increased slightly to $8.8 \pm 0.3458$ from the baseline. In addition, immunizations and challenge of rats in the UV inactivated group resulted in a statistically significant $(p<0.05)$ increase in mean white blood cell counts compared to both baseline counts and the positive control group. This is because the immunized rats recorded relatively higher mean WBC counts of $10.58 \pm$ 0.7702 . However, rats in the positive control group had decreased WBC counts of $5.12 \pm 0.3385$ which is the lowest when compared to all groups. Statistical analysis shows a significant difference $(\mathrm{P}<0.05)$ in WBC counts of rats in the immunized and positive control groups.

\section{Lymphocytes Profile of Rats Immunized and Controls}

Albino rats in all the three groups recorded an increase in mean lymphocyte counts after 38 days as follows: UV inactivated (73.18\%), Negative control (66.32\%) and Positive control (57.06\%) (Fig. 3). Although the increase in lymphocyte counts relative to the baseline counts $(67.42 \%)$ in all the groups was not significant $(\mathrm{P}>0.05)$, the mean lymphocyte counts of rats in the vaccinated group shows a significant difference $(\mathrm{P}<0.05)$ when compared to rats in the control groups after 38 days. Similarly, there was a significant difference $(\mathrm{P}<0.05)$ in lymphocyte counts when negative and positive control groups were compared. However, mean lymphocyte counts of rats in positive control group at the end of the experiment $(57.06 \%)$ shows a significant decrease when compared with the baseline counts.

\section{Granulocytes Profile of Immunized Rats and Controls}

The mean granulocytes counts of immunized and control groups is presented in Fig. 4. The mean percentage granulocyte counts of rats after acclimatization (baseline) in all the groups was $25.94 \pm 1.061$. At day 38 , rats in the negative control group recorded a statistically insignificant slight increase $(\mathrm{P}>0.05)$ in the level of granulocytes to $25.94 \pm 1.061$. However, rats in UV inactivated and positive control groups recorded significantly lower $(\mathrm{P}>0.05)$ granulocyte counts of $19.88 \%$ and $15.7 \%$ respectively as compared to the baseline counts. 


\section{Mid-Range Cells (MEB) Profile of Immunized Rats and Controls}

The result of percentage Mid-range cells (monocytes, eosinophils and basophols) for immunized and control albino rats after acclimatization and subsequent treatment is presented in Fig. 5. The mean percentage of MEB for rats in all groups after 7 days of acclimatization (baseline) was 3.66 \pm 0.16 . With the exception of rats in the positive control group that recorded a statistically significant $(\mathrm{P}<0.05)$ MEB of $27.28 \%$ at day 38 , rats in UV inactivated and negative control groups recorded lower MEB of 5.82 and $4.32 \%$ respectively. Although the slight increase in MEB at day 38 in the two groups (negative control and UV inactivated groups) were not statistically significant $(\mathrm{P}<0.05)$ from the baseline counts and between UV inactivated and negative control groups at day 38 , there was a statistically significant difference
$(\mathrm{P}<0.05)$ in MEB recorded when compared to positive control group.

\section{Survival Analysis}

The survival result of the rats immunized with UV killed $C$. albicans and the controls after 28 days is presented in Table 1 and Fig. 6. No death was recorded in rats from negative control and Heat inactivated groups during the four weeks of observation. However, 75 and 25 percent of rats in the positive control group died in the first and second weeks respectively.

Table 1: Percentage mortality rate of rats immunized with Heat inactivated and control groups

\begin{tabular}{lccll}
\hline Vaccination & \multicolumn{2}{c}{-- \% Survival -- } & & \\
Status & Week 1 & Week 2 & Week 3 & Week 4 \\
\hline Heat group & 0.0 & 0.0 & 0.0 & 0.0 \\
Negative control & 0.0 & 0.0 & 0.0 & 0.0 \\
Positive control & 75.0 & 25.0 & 0.0 & 0.0 \\
\hline
\end{tabular}

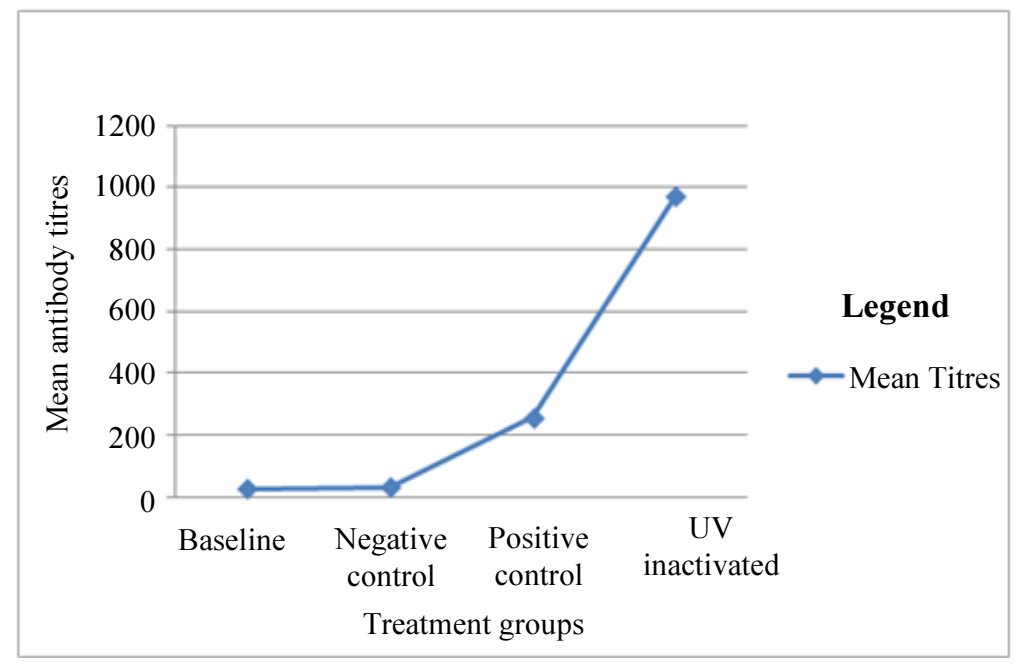

Fig. 1: Antibody titers of rats vaccinated with inactivated C. albicans and controls

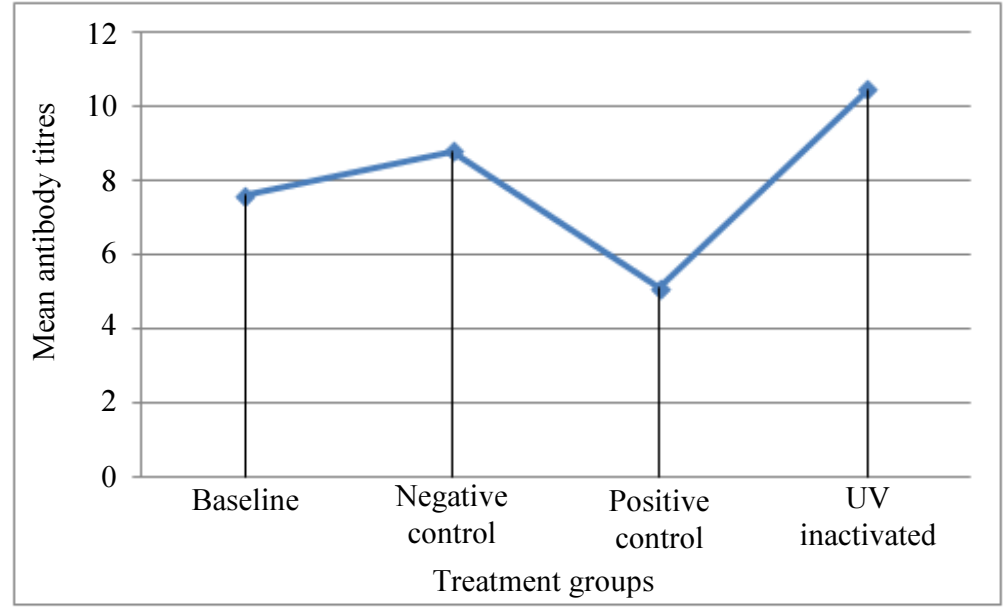

Fig. 2: White blood cell count of rats vaccinated with inactivated C. albicans and controls 


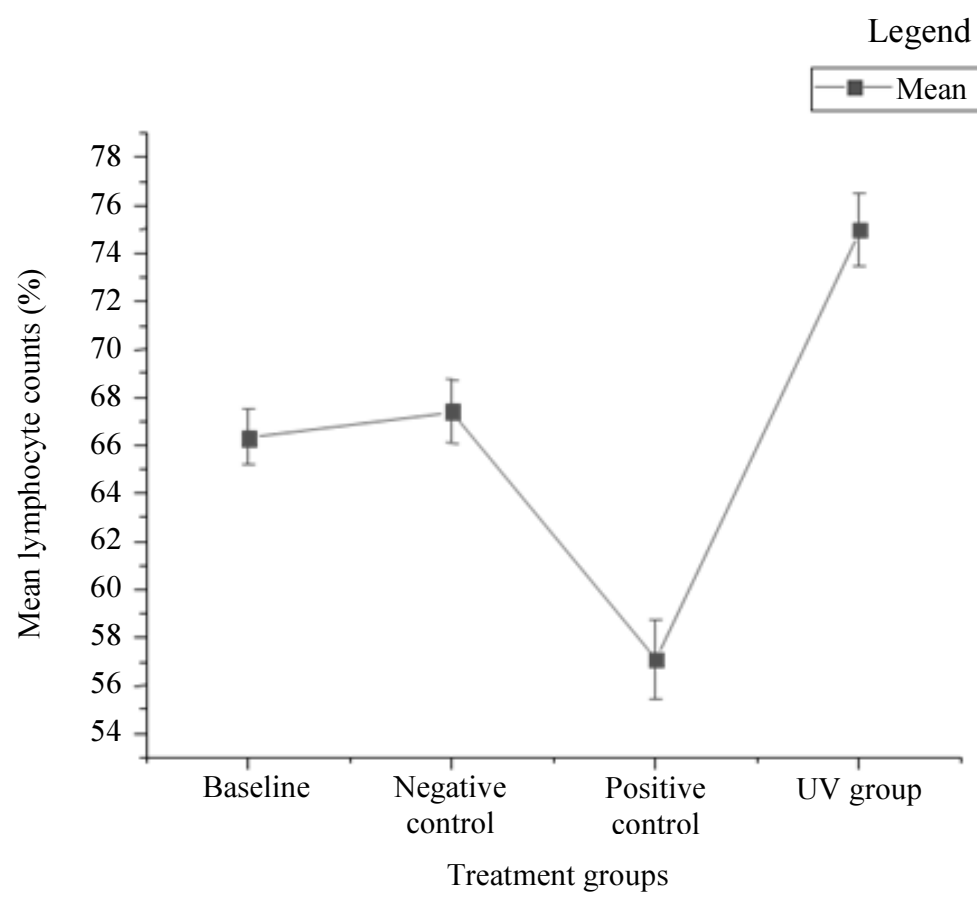

Fig. 3: Lymphocytes Profile of Albino Rats Vaccinated with UV killed C. albicans

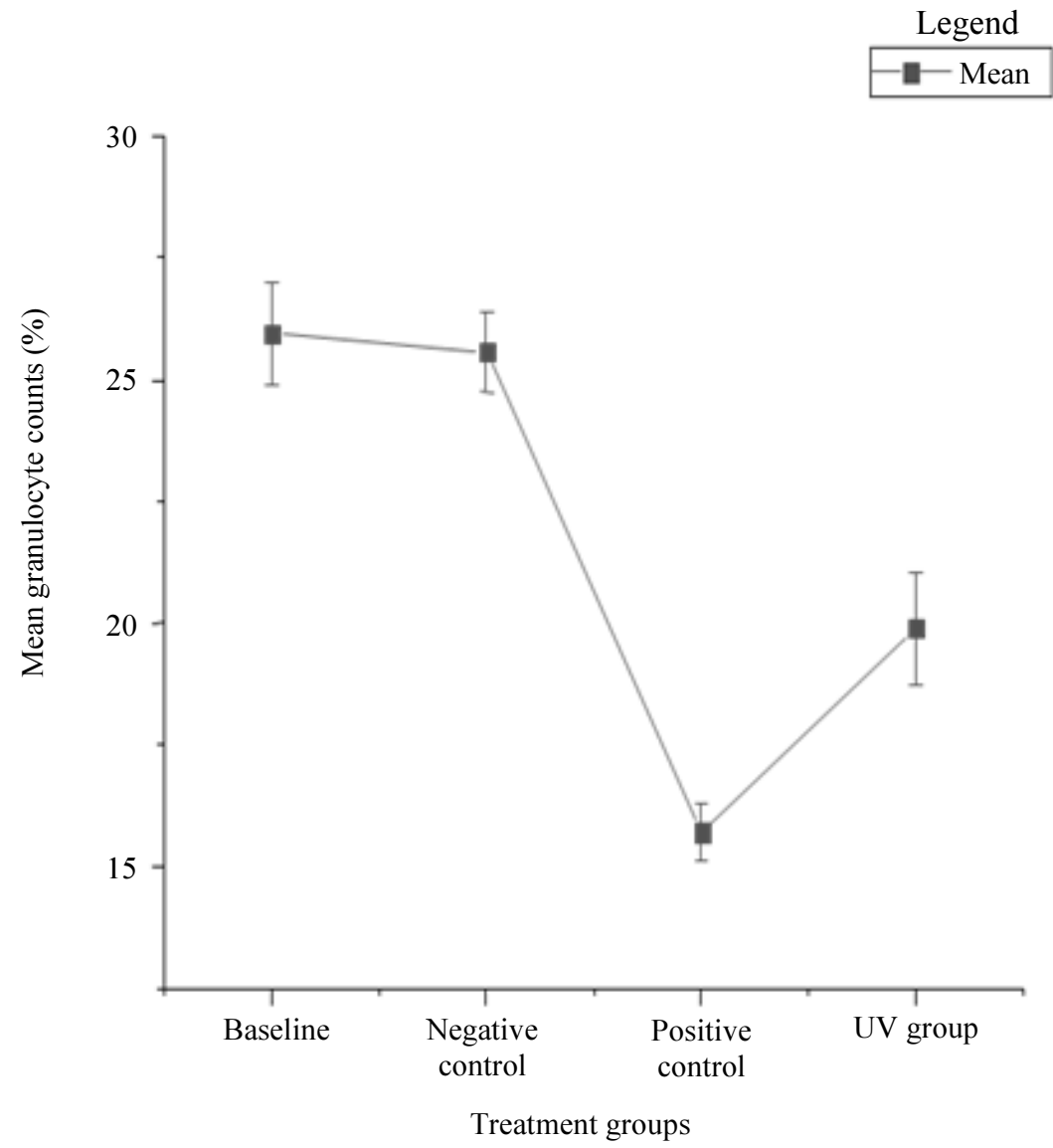

Fig. 4: Granulocytes Profile of Rats Vaccinated with killed C. albicans and Control 


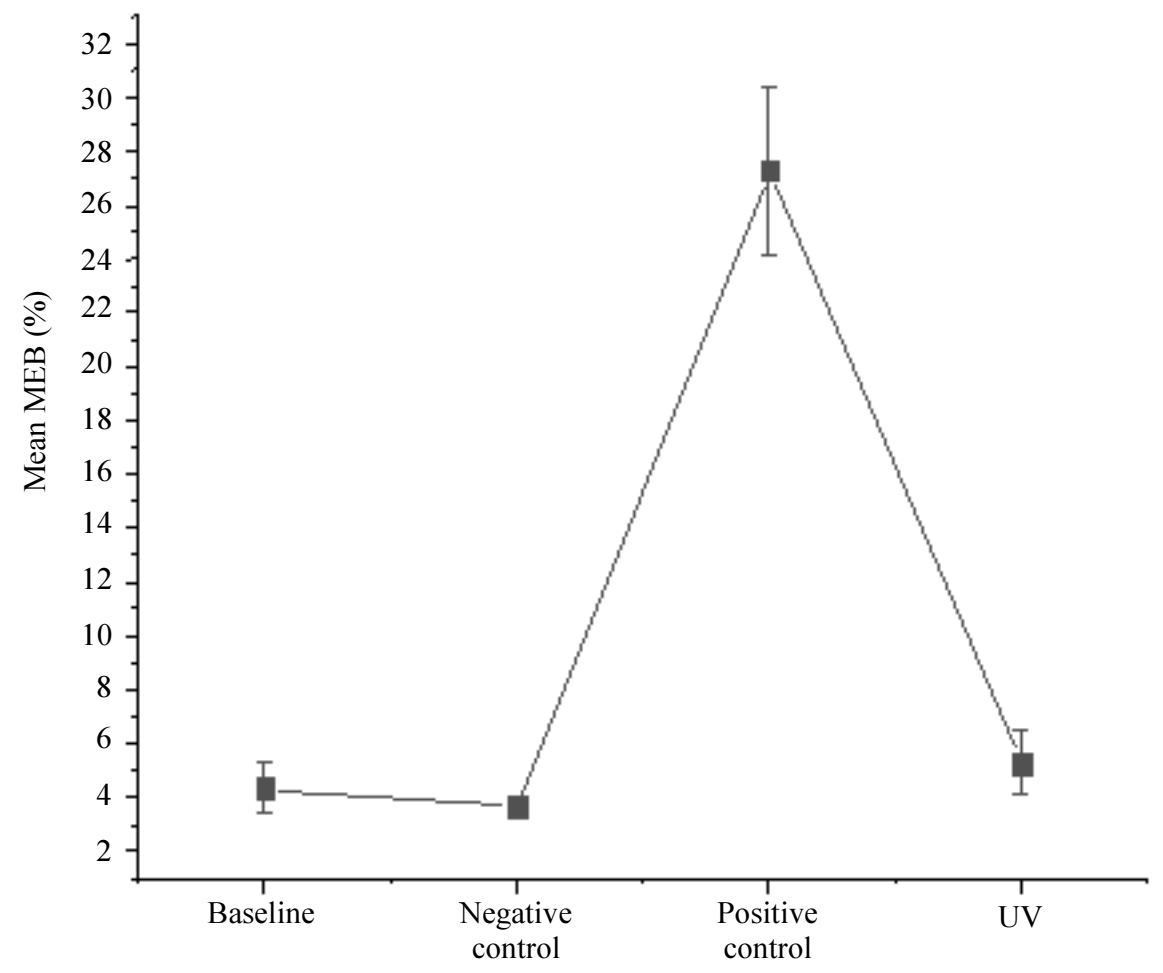

Treatment groups

Fig. 5: Mid-Range cells (MEB) profile of rats vaccinated with inactivated C. albicans and controls

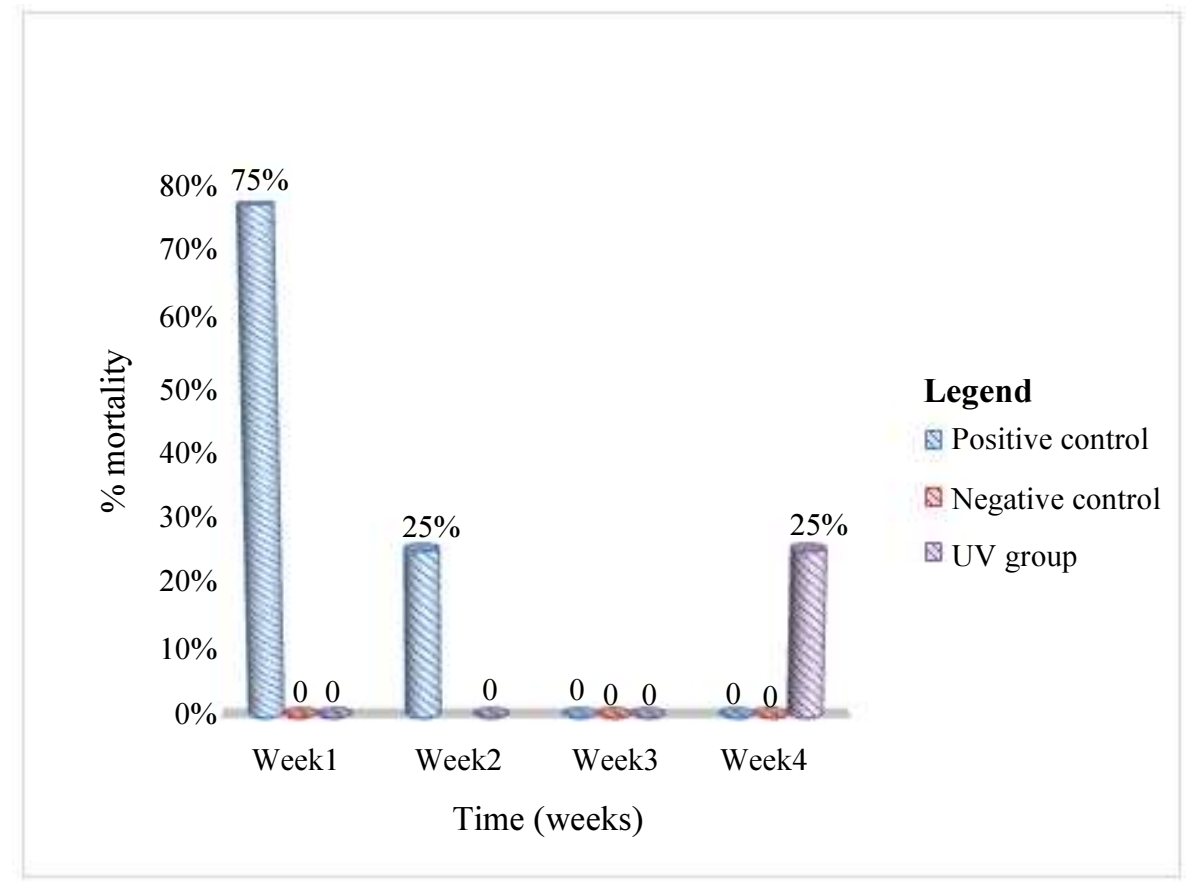

Fig. 6: Percentage mortality of rats immunized with UV inactivated C. albicans and Control groups 


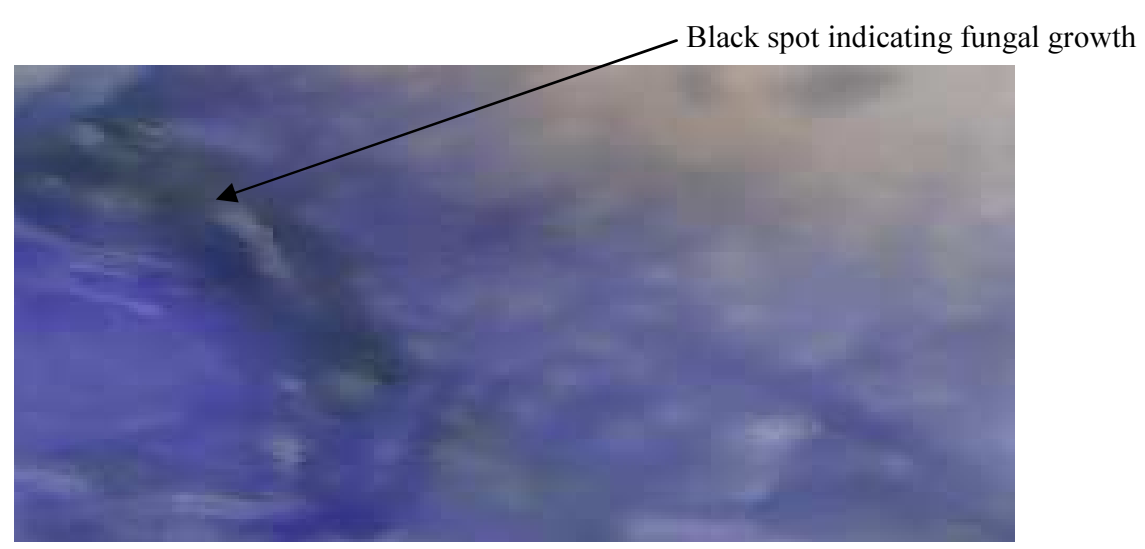

Fig. 7: Sectioned and stained kidney of fungal infected albino rat (X500 magnification)

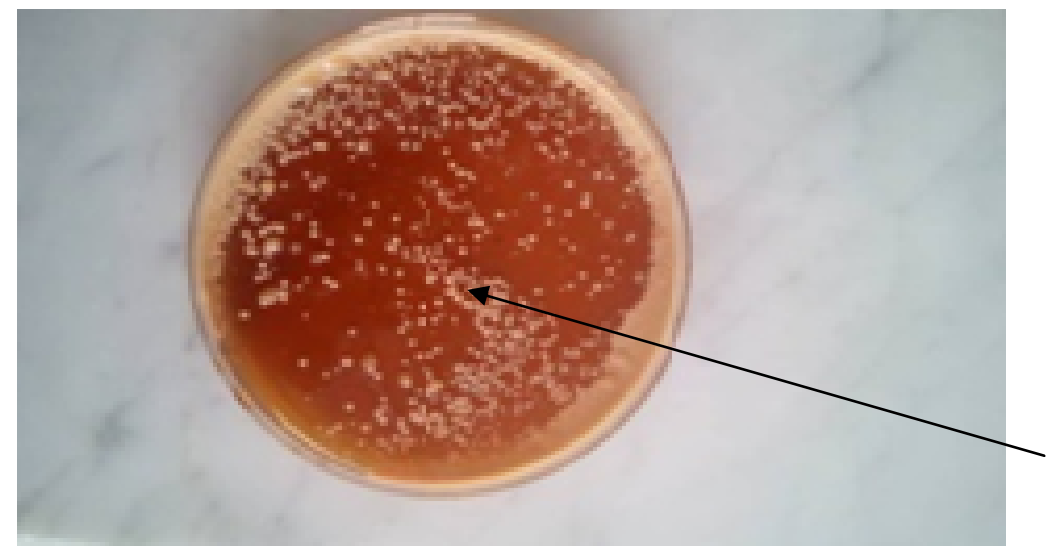

White creamy spot of C. albicanon SDA

Fig. 8: Fungal growth of homogenized and cultured kidney

\section{Kidney Fungal Burden}

The fungal burden in kidney of dead rats is presented in Fig. 7, as black spots in the sectioned and stained kidneys of rats. In addition, fungal (C. albicans) growth was observed when homogenized kidneys of dead rats were cultured on SDA plates (Fig. 8).

\section{Discussion}

The significant increase of mean percentage granulocytes in the immunized groups in response to the challenge shows the roles of white blood cells in the fight against Candida albicans infections. This is because granulocytes, macrophages and monocytes are the first line defense mechanism recruited in the first few days of infection to engulf, digest and present pathogens for specific immune response (Aderem, 2003). This increase in granulocytes for immunized albino rats is in line with the findings of Gow et al.(2007) that reported significant production of cytokines and chemokines which have phagocyte stimulatory properties.
However, the observable decrease of granulocytes in the infected group (positive control) is an indication of immunosuppression. This could be as a result of the ability of C. albicans to undergo switching or morphogenesis. These properties allow C. albicans to escape phagocytosis by piercing and subsequent killing of phagocytic cells leading to a decrease in circulating granulocytes (Uwamahoro et al., 2014). This finding is not in conformity with a study conducted by Jamalzadeh et al.(2009) which reported an increase in total white blood cells (neutrophils, basophils and eosinophils) in fungal infection of Caspian salmon.

The mid-range cells comprised of monocytes, eosinophils, basophils and immature cells (Abacus 380 automated blood analyzer manual). Therefore, the increase in mean percentage of mid-range cells in positive control as compared to the immunized and negative control groups may be attributed to the presence of increased amount of $C$. albicans in the blood to meet up with the burden. Therefore, could be because there is a continuous proliferation of immature cells in circulation (left shift). This indicates that neutrophil 
consumption in the tissues surpasses supply in the bone marrow (Honder et al., 2016).

Moreover, the significant increase in the percentage of lymphocytes in the immunized groups when compared to both controls could be because after immunization, the rats became sensitized and this result to the stimulation of memory cells to produce a substantial amount of lymphocytes (Delvis, 2014). The increase in lymphocytes is in conformity with a study conducted by Jamalzadeh et al.(2009) which showed a significant decrease in fungal infection.

Clinical observations reported by Mathews and Burnie, (2001) indicated that antibodies play an important role in host defense against disseminated candidiasis because individuals with defects in cell mediated immune response are particularly prone to superficial but not disseminated candidiasis. Similarly, the significant $(\mathrm{P}<0.05)$ increase in the level of antibodies of the immunized rats shows that inactivated C. albicans has the potential of stimulating humoral immunity as evident in this study. The UV group produced a mean antibody titers of $972.8 \mu \mathrm{g} / \mathrm{ml}$. This could be as a result of recognition of the immunogenic proteins and glycoproteins on the cell surface and subsequent stimulation of memory cells to produce significant quantity of antibodies on a second encounter of similar antigens (Delvis, 2014). Thus, this study correlates with findings of Cárdenas-Freytaget al. (1999) which reported a significant production of $3200 \mu \mathrm{g} / \mathrm{ml}$ immunoglobulins that were immunoprotective against candida infections in mice immunized with heat killed $C$. albicans in combination with adjuvant. Similarly, Thomas et al. (2006) also indicated a significant production of antibodies that are immune-protective during infection. In another study by Evron (1980), the circulating antibodies in immunized mice that were immunoprotective were greater than $256 \mu \mathrm{g} / \mathrm{ml}$. Furthermore, in two separate studies conducted by Saville et al. (2008; 2009), showed vaccination with live attenuated $C$. albicans in mice challenged with a lethal dose of $5.2 \times 10^{6}$ virulent cells has a protective effect on candida infections. It is also interesting to note that UV inactivated $C$. albicans produced more antibodies, lymphocytes and granulocytes as compared to the positive control. This could not be unconnected with the fact that $\beta$-glucans of live cells are normally masked by mannan-mannoprotein layer thus precluding recognition. However, the $\beta$-glucans of UV inactivated $C$. albicans is exposed to recognition (Gow et al., 2007). This finding is in agreement with Gow et al. (2007) who reported that heat inactivated $C$. albicans induced significantly greater level of cytokines and chemokines which are known activators of immune cells.

There is a $75 \%$ survival of rats in UV inactivated group as compared to the un-immunized infected rats where $100 \%$ death was recorded before the end of the experiment. This agrees with the report of Thomas et al.
(2006). The mortality was caused probably by the breakdown in osmotic balance when the tissues were destroyed by the penetration of the hyphae and the lethargy that resulted from excessive energy exerted to overcome infection stress. Therefore, this result is also in agreement with the research conducted by Shah (2002).

\section{Conclusion}

The findings of this study showed that heat inactivated C. albicans elicited significant immunological response in immunized albino rats. The study also clearly indicated an interesting immuneprotection of the immunized rats against $C$. albicans infections. It was also shown that $C$. albicans infections or systemic candidiasis can cause immune-suppression, morbidity and even mortality as in the case of rats in the positive control group.

\section{Recommendations}

Further study should be conducted on different doses of UV on freshly isolated $C$. albicans that will elicit substantial amount of immunological response in rats.

\section{Acknowledgement}

I want to acknowledge the effort the staff of Department of Microbiology and Biotechnology of National Institute of Pharmaceutical Research and Development, Abuja for his guidance in the course of the experinment.

\section{Author's Contributions}

We participated in the conception, experinment and write up of this paper

\section{Conflict of Interest}

We declare that there is no conflict of interest.

\section{References}

Aderem, A.,2003. Phagocytosis and the inflammatory response. J. Infect. Dis., 187: 340-345. DOI: $10.1086 / 374747$

Bin-Hafeez, B., I. Ahmad, R. Haque and S. Raisuddin, 2001. Protective effect of Cassia occidentalis on cyclophosphamide - induced suppression of humoral immunity in mice. J. Ethnopharmacol., 75: 13-18. PMID: 11282437

Cárdenas-Freytag, L., E. Cheng, P. Mayeux, J.E. Domer and J.D. Clements, 1999. Effectiveness of a vaccine composed of heat-killedcandida albicans and a novel mucosal adjuvant, LT (192G), against systemic candidiasis. Infect. Immunity J., 67: 826-833. PMID: 9916097 
CDC, 2016. Fungal diseases (Candidiasis). USDepartment of Health and Human Services.

CDC, 2015. Vulvovaginal candidiasis.Sexually Transmitted Disease Treatment Guidelines.

Cooke, M.V., R.J. Miles, R.G. Price, G. Midgley and A.C. Richardson, 2002. New chromogenic Agar medium for the identification of Candida spp. Applied Environ. Microbiol., 68: 3622-3627. DOI: 10.1128/AEM.68.7.3622-3627.2002

Delvis, P.J., 2014. The Innate and Adaptive Responses. In: The Autoimmune Diseases, Noel, R.R. and I.R. Mackay (Eds.), Science Direct. pp: 53-68.

Elmer, W.K., D.A. Stephen and M.J. William, 1992. Laboratory approach to the diagnosis of fungal infections. 14th Edn., J.P. Lipincott company. Philadelphia, pp: 387-840.

Evron, R., 1980. In Vitrophagocytosis of candida albicans by peritoneal mouse macrophages. Infect.Immunity, 28: 963-971. PMID: 6995329

Gow, N.A. and B. Hube, 2012.Importance of C. albicans cell wall during commensalism and infection. Current Opin. Microbiol., 15: 406-412. DOI: 10.1016/j.mib.2012.04.005

Gow, N.A., M.G. Netea, C.A. Munro, G. Ferwerda and S. Bates et al., 2007. Immune recognition of Candida albicans $\beta$-glucan by Dectin-1. J. Infect. Dis. 196: 1565-1571. DOI: 10.1086/523110

Honder, T., T. Uehara, G. Matsumoto, S. Arai and M. Sugano, 2016. Neutrophil left shift and white blood cell count as markers of bacterial.Infect.Clin. Chimica Acta, 457: 46-53.

DOI: 10.1016/J.CCA.2016.03.017

Jamalzadeh, H.R., A. Kesvan, M.R. Ghomi and F. Gherardi, 2009. Comparison of blood indices in healthy and fungal infected Caspian Salmon. Africa J. Biotechnol., 8: 319-22.

Lam, P., S.H.L. Kok, K.K.H. Lee, K.H. Lam and D.K.P. Hauet al., 2016. Sensitization of Candida albicans to terbinafine by berberine and berberrubine. Biomed. Reports, 4: 449-452. DOI: $10.3892 /$ br.2016.608

Leleu, G., P. Aegerter and B. Guidet, 2002. Systemic candidiasis in intensive care units: A multicenter, matched-cohort study. J. Critical Care, 17: 168-175. PMID: 12297992

Lopez-Ribota, J.L., M.B. Casanova, A.C. Murgui and J.P. Martınez, 2004. Antibody response to Candida albicans cell wall antigens. FEMS Immunol. Med. Microbiol., 41: 187-196.

Matthews, R. and J. Burnie, 2001. Antifungal antibodies: A new approach to the treatment of systemic candidiasis. Current Opinion Investigational Drugs, 2: 472-476.

Ortega, M., F. Marco, A. Soriano, M. Chen, S.C., E.G. Playford and T.C. Sorrell, 2010. Antifungal therapy in invasive fungal infections. Current Opin. Pharmacol., 10: 522-530.
Pereira, C.A., R.L. Romeiro, A.C. Costa, A.K. Machado, J.C. Junqueira et al., 2011. Susceptibility of Candida albicans, staphylococcus aureus and streptococcus mutans biofilms to photodynamic inactivation: An in vitro study. Lasers Med. Sci., 26: 341-348. DOI: 10.1007/s10103-010-0852-3

Saville, S.P., A.L. Lazzell, A.K. Chaturvedi, C. Monteagudo and J.L. Lopez-Ribot, 2008. Use of a genetically engineered strain to evaluate the pathogenic potential of yeast cell and filamentous forms during Candida albicans systemic infection in immunodeficient mice. Infect. Immunity, 76: 97-102. DOI: 10.1128/IAI.00982-07

Saville, S.P., A.L. Lazzell, A.K. Chaturvedi, C. Monteagudo and J.L. Lopez-Ribot, 2009. Efficacy of a genetically engineered Candida albicans tetNRG1 strain as an experimental live attenuated vaccine against hematogenously disseminated candidiasis. Clin. Vaccine Immunol., 16: 430-432. DOI: 10.1128/CVI.00480-08

Shah, V., E. Bayeta and B.H.S. Lau, 2002. Pyenogenol augments macrophage phagocytosis and cytokine secretion. Pakistan J. Tabriz University Medical Sci., 1: 196-201.

Staib, P. and J. Morschhäuser, 2007. Chlamydospore formation in Candida albicans and Candida dubliniensis an enigmatic developmental programme. Mycoses, 50: 1-12.

DOI: $10.1111 /$ j.1439-0507.2006.01308.x

Spellberg, B., K. Marr and S.G. Filler, 2012. Candida: What should Clinicians and Scientists be Talking About? In: Candida and Candidiasis, Calderone, R.A. and C.J. Clancy (Ed.), ASM Press, pp: 225-242.

Tada, R., J.P. Latgé and V. Aimanianda, 2013. Undressing the fungal cell wall/cell membrane the antifungal drug targets. Current Pharmaceutical Des., 19: 3738-3747.

Thomas, D.P., A. Viudes, C. Monteagudo, A.L. Lazzell and S.P. Saville et al., 2006. A proteomic-based approach for the identification of Candida albicans protein components present in a subunit vaccine that protects against disseminated candidiasis. Proteomics, 6: 6033-6041.

DOI: $10.1002 /$ pmic. 200600321

Uwamahoro, N., J. Verma-Gaur, H.H. Shen, Y. Qu and R. Lewis et al., 2014. The pathogen C. albicans hijacks pyroptosis for escape from macrophages. Am. Society Microbiology, 5: 1-11.

Wang, X., X.L.E. Sui and Y. Jiang, 2015.Vaccines in the treatment of invasive landdiseases. Virulence, 6: 309-315. DOI: 10.4161/21505594.2014.983015 